\title{
TÉCNICAS DE GEOPROCESSAMENTO E DE AMOSTRAGEM PARA O MAPEAMENTO DE ATRIBUTOS ANISOTRÓPICOS DO SOLO ${ }^{(1)}$
}

\author{
M. M. VALERIANO ${ }^{(2)} \&$ H. PRADO(2)
}

\begin{abstract}
RESUMO
Este trabalho abordou a utilização de geoestatística para a otimização de esquemas de amostragem, concentrando-se especialmente no impacto da anisotropia na estruturação geométrica do desenho experimental e no processo de geração de imagens contínuas. 0 atributo estudado foi o teor de argila na camada superficial do solo, levantada em novembro de 1998, em área de Latossolo Vermelho-Escuro, localizada no município de Campos Novos Paulista (SP). A variabilidade espacial e a ani sotropia do teor de argila foram determinadas por meio do exame dos semi variogramas obtidos, os quais foram ajustados a modelos teóricos para a obtenção de seus parâmetros (tipo de modelo de semi variograma, pepita, patamar e alcance). 0 fator de anisotropia observado implicou uma configuração oval, ou anisotrópica, da área de influência dos dados amostrais, em conformidade com a estrutura da variabilidade espacial observada. A anisotropia foi atribuída a fatores intrínsecos do solo, condicionados pela topografia do respectivo processo de formação, e extrínsecos, tais como: a mecanização, divisão de glebas e demais práticas de manejo, feitas no sentido das linhas de plantio, transversalmente ao gradiente topográfico. Considerando essas dimensões, a área de representatividade das amostras (ha/amostra) variou de um esquema a outro, modificando, assim, a densidade de amostras requerida para cada nível de variância. A proporção entre estas áreas mostrou o impacto da anisotropia sobre o número de amostras requerido para cada nível de variância desejável.
\end{abstract}

Termos de indexação: SI G-Sistemas de Informação Geográfica, imagem digital, interpolação de dados tabulares, teor de argila, LatossoloVermelho-E scuro.

(1) Recebido para publicação em junho de 2000 e aprovado em março de 2001.

(2) Pesquisador do Centro de Sol os e Recursos Agroambientais do InstitutoAgronômico de Campinas - IAC. Av. Barão de I tapura 1481, Caixa Postal 28, CEP 13001-970 Campinas (SP). E-mail: valerian@barao.iac.br; hprado@barao.iac.br 


\title{
SUMMARY: GEOPROCESSING AND SAMPLING TECHNIQUES FOR ANISOTROPIC SOIL ATTRIBUTES MAPPING
}

\begin{abstract}
This study presents the use of geostatistical analyses for the design of sampling schemes, focusing specially on theani sotropy impacts on the dimensioning of sample units and image generation techniques. The attribute studied was the superficial clay content, measured during November 1998 in a Typic Hapludox, in Campos Novos Paulista (SP). Spatial variability and anisotropy of clay content were determined by examining the semivariograms, which were fit to theoretical models to obtain its parameters (variogram model type, nugget, sill and range). The observed anisotropy factor expressed an oval, or anisotropic, configuration of the influence area related to the sampling points, according to the structure detected for the spatial variability. The ani sotropy could be explained by soil intrinsic factors, conditioned by thetopography in theformation processes, and extrinsic factors, such as mechanization, partiti oning terraces and other managing practices, which are applied in plough lines, transverse to topographic gradient. These dimensions caused the variation of the area of samples representativeness (ha/ samples) between thesampling designs, modifying the required density for a given variance level. The ratio of these areas showed the impact of anisotropy on the number of samples required for each desirable level of variance.
\end{abstract}

Index terms: GIS-Geographical I nformation Systems, digital image, interpolation of tabular data, clay content, Typic Hapludox.

\section{INTRODUÇÃO}

A representação de um fenômeno geoambiental da paisagem por meio de polígonos de homogeneidade, a exemplo dos mapas de solo, é muitas vezes inadequada, porque as mudanças espaciais não ocorrem em locais definidos, com limites abruptos. Além disso, limites estabelecidos a partir de critérios sobre um atributo podem não ser adequados para representar a variação de outros. As principais limitações do mapeamento por polígonos "uniformes" advêm de não se considerar a possibilidade detransições graduais, ou tendências, na unidade de paisagem, bem como por se ignorar o fenômeno da autocovariância, pelo qual se espera que pontos mais próximos apresentem valores similares de atributo, em contraposição com os mais distanciados. Um modo comum de observar a variação espacial dentro de unidades de mapeamento é a segmentação da área em escalas mai ores (repetindo o princípio dos polígonos), porém com subdivisões espaciais bem reduzidas. 0 tratamento é feito então sobre uma base de dados numa estrutura espacial conhecida como grade regular, raster, ou imagem.

Muitas vezes, a variação espacial de atributos ambientais ocorre continuamente, de modo a impossibilitar uma descrição por simples regressões polinomiais, como em análises de tendência. Há, além desta estrutura de variação, uma distribuição al eatória de resíduos, porém correlacionados, na escala de observação de cada ponto e suas imediações. Este raciocínio implica em um modelo de variabilidade espacial com três componentes, começando pela média ou pela tendência do atributo na unidade de mapeamento, definida conforme suas particularidades fisiográficas ou ecológicas. Sobreposta a esta primeira estrutura, há um tipo de variação, correlacionada espacialmente, que não pode ser identificada como uma componente determinística da paisagem. Enfim, há uma terceira componente que consiste essencialmente em variação al eatória não correl acionada, que pode ser explicada por erros experimentais ou por variações espaciais em escalas incompatíveis com o desenho de amostragem praticado. Variáveis descritas com estas três componentes são referidas por variáveis regionalizadas (Burrough, 1987).

A análise da variabilidade espacial de um atributo concentra-se na segunda e na terceira componente estrutural da variação, indicando que algumas premissas devem ser observadas. A mais importante delas determina que as propriedades estatísticas da variação espacial não correlacionada sejam constantes por toda a unidade mapeada. Esta é conhecida como a hi pótese da estacionaridade estatística, sem a qual não se poderiam aplicar métodos estatísticos para análises espaciais (Vieira, 1995).

As técnicas da geoestatística lidam com problemas de espacialização de variáveis e representam uma promissora ferramenta para trabalhos em Sistema de I nformação Geográfica-SI G (Oliver \& Webster, 1990), em três aplicações básicas: (a) estimativas: para inferir atributos em pontos diferentes daqueles originais, isto é, onde estes não foram coletados; (b) previsões: para detectar 
tendências e locais de máximos e mínimos; (c) desenho de experimentos: para otimizar a segmentação da área em unidades de espaço.

Com efeito, cada vez mais, em trabalhos relativamente recentes, análises da variabilidade ou da dependência espacial vêm sendo incorporadas em métodos de preparação de dados ambientais, visando ao geoprocessamento (Englund \& Sparks, 1988; Oliver \& Webster, 1990; Legendre, 1993; Atkinson, 1996; Salviano et al., 1998). As técnicas de geoestatística são reconhecidas por estes autores como adequadas nos estudos de variabilidade espacial, por permitir a escol ha da krigagem como método de interpolação, quando aplicada. Várias utilizações da geoestatística em mapeamento vêm sendo demonstradas, destacando-se aqui o dimensionamento de grades experimentais (Atkinson, 1996, Gonçalves et al., 1999). A técnica da krigagem minimiza o erro (ou variância) associado a cada estimativa. Além da estimativa do valor da variável, a técnica possibilita a estimativa da variância amostral para cada ponto. I sto permite a seleção de esquemas de amostragem para o mapeamento ótimo de variáveis espacializadas.

Os semi variogramas são representações gráficas da função de dependência em relação à distância, através das quais podem-seajustar model os teóricos de variabilidade espacial. Os semivariogramas experimentais são estimados pela seguinte equação:

$$
\gamma(\mathrm{h})=\left(\sum_{\mathrm{i}=1}^{\mathrm{N}(\mathrm{h})}\left[\mathrm{Z}\left(\mathrm{x}_{\mathrm{i}}\right)-\mathrm{Z}\left(\mathrm{x}_{\mathrm{i}}+\mathrm{h}\right)\right]^{2}\right) / 2 \mathrm{~N}(\mathrm{~h})
$$

\section{em que}

$N(h)$ é o número de pares de valores $Z\left(x_{i}\right)$ e $Z\left(x_{i}+h\right)$ medidos, separados por um vetor $h$. A cada par estão associadas, além do módulo de h, a diferença de val or do atributo medido e a direção do vetor $h$. O semivariograma apresenta-se, em geral, como um gráfico crescente, pela provável semelhança entre pontos a menores distâncias. Ao semivariograma experimental, calculado a partir das observações, associam-se model os teóricos de semivariogramas, definidos pelo tipo de modelo (linear, esférico, gaussiano, exponencial, potência) epel os parâmetros numéricos efeito pepita, patamar e alcance. $\mathrm{O}$ alcance é indicado pela distância a partir da qual cessa oincremento da variância estimada, queatinge um valor de patamar. Representa o alcance da dependência espacial, ou raio de influência, dos pontos amostrados sobre sua vizinhança. Os procedimentos de levantamento e interpolação de dados ter restres devem ser feitos com espaçamentos menores do que com o al cance obti do para a variável estudada, quando o objetivo émapeamento. Por outro lado, quando se quer caracterizar uma área em função da média de amostras, independentes entre si, devem-se utilizar espaçamentos maiores do que o alcance, para evitar redundância (Vieira, 1995).
O patamar do semivariograma corresponde teoricamente à variância máxima dos dados. Os dados ajustados ao modelo linear (sem patamar) indicam que o conjunto todo foi col etado dentro de uma área de dimensões menores do que o alcance. O semivariograma não intercepta obrigatoriamente a origem do gráfico, havendo uma variância inicial da curva obtida. O efeito pepita é a estimativa de semivariância para a distância nula, indicado pelo valor da ordenada (eixoy) na intersecção com a curva. Corresponde à componente aleatória da variabilidade e indica a ocorrência de alterações abruptas ao longo da superfície de variação do dado analisado (Burrough, 1987). Portanto, dados com el evado efeito pepita requerem maior cuidado para sua espacialização e mapeamento (Valeriano, 1999).

A otimização dos esquemas de amostragem pode ser baseada na curva de semivariograma obtida, consi derando o estabel ecimento de níveis aceitáveis de variância de estimativa associada aos espaçamentos. Este critério pode ser estabelecido pel o operador, conforme os objetivos da amostragem praticada. Podem ser definidos dois grupos distintos de interesse: primeiro, representado pela pesquisa que deseja obter amostras independentes entre si, para caracterizar, por exemplo, uma área por meio da média do atributo observado; o segundo, representado pela pesquisa que objetiva executar uma interpolação dos dados. Para o último caso, é desejável que haja uma dependência entreamostras vizinhas, objetivando minimizar a variância da estimativa, o que é uma premissa fundamental, implícita no processo de interpolação.

Muitos trabalhos avaliaram a variabilidade espacial deatributos do solo, com vistas em levantar informações que subsidiem o planejamento de experimentos (Albuquerque et al., 1996; Souza et al., 1997, 1998; Salviano et al., 1998; Carval hoet al., 1998).

Quando a variabilidade espacial dos dados ocorrer da mesma maneira em todas as direções, o comportamento é chamado de isotrópico. Se não, é chamado anisotrópico. A isotropia ocorre em situações em que não há um controle direcional atuando na forma do padrão espacial. Pode-se esperar isotropia, por exemplo, da textura do solo originado do granito intemperizado, uma vez que este material varia uniformemente em diferentes direções. A análise geoestatística pode ser feita, restringindo-se direções no espaço geográfico, para determinar a estruturação geométrica da variabilidade do fenômeno, ou seja, a anisotropia. I sto é feito selecionando-se a direção dos vetores $h$, associada aos pares $Z\left(x_{i}\right)-Z\left(x_{i}+h\right)$.

Muitos dos fenômenos naturais apresentam variações que são maiores em uma direção do que em outras. Podem-se exemplificar tipicamente os atributos dos sol os quando descritos ao longo de uma toposseqüência, o que pressupõe rel ativa estabilidade dos atributos na direção ortogonal. 
O presente trabalho abordou a utilização de geoestatística para o dimensionamento de unidades amostrais, concentrando-se especialmente no impacto da anisotropia na estruturação geométrica do desenho experimental e no processo de geração de imagens contínuas.

\section{MATERIAL E MÉTODOS}

O experimento foi realizado em uma área situada no município de Campos Novos Paulista (SP), com coordenadas aproximadas $22^{\circ} 35^{\prime} \mathrm{S}$ e $50^{\circ} 00^{\prime} \mathrm{W}$ Gr. e altitude de $500 \mathrm{~m}$. O clima local enquadra-se na classe Aw da classificação de Köppen. $\mathrm{Na}$ área estudada, predomina o Latossol o Vermel ho-E scuro álico A moderado textura média (Camargo et al., 1987), o qual se enquadra atual mente como L atossol o Vermel ho distrófico típicoálico A moderadotextura média (EMBRAPA, 1999), no nível de família. De acordo com a classificação americana, classifica-se no nível de subgrupo como Typic Hapludox (Soil Survey Staff, 1990). E m al guns pontos (cerca de 8\%), o solo foi classificado como Latossolo Vermel hoEscuro distrófico A moderado textura média (na classificação atual, Latossolo Vermelho distrófico típico álico A moderado textura média), por apresentar saturação por alumínio inferior a 50\%. O relevo é suavemente ondulado, com declividade média de aproximadamente $0,02 \mathrm{~m} \mathrm{~m}^{-1}$ a $0,03 \mathrm{~m} \mathrm{~m}^{-1}$. A orientação da vertente (direção de dedive) na área estudada é de $51^{\circ}$ de azimute.

As amostras desol o foram retiradas nos 92 pontos de uma malha regular com espaçamento de $50 \mathrm{~m}$, estabelecida no campo com auxílio de técnicas de localização por sistema de posicionamento global ("gl obal positioning system"-GPS) diferencial. O sol o foi examinado em cada um dos pontos de grade, mediante tradagem, nas profundidades de 0-20, 2040, 40-60 e 60-100 cm. O trabal ho de campo foi feito em 16 de novembro de 1998.

E mbora tenham sido feitas descrições completas dos perfis de sol o amostrados em campo, as análises deste trabal ho ficaram restritas ao teor de argila na camada superficial do solo (0-20 cm deprofundidade), obtida pelo método da pipeta em material disperso com hexametafosfato de sódio e hidróxido de sódio (Camargo et al., 1986). Segundo Burroughs (1987), o teor deargila do solo ilustra com clareza a ani sotropia e a variabilidade espacial em escalas diversas.

Os resultados foram processados, após digitação de arquivos em planilhas eletrônicas, com referenciação geográfica em metros, relativa ao canto inferior esquerdo (sudoeste) do retângulo envol vente da área, cujas dimensões aproximadas foram de 1.000 m (emy) e900 m (em x). A análisegeoestatística foi aplicada de modo isotrópico e anisotrópico, por meio do programa VARIOWIN (Pannatier, 1996). A análise anisotrópica foi orientada nas direções correspondentes à linha de plantio (141-3210) e à sua transversal (51-2310). Foi definida uma tolerância angular de $22,5^{\circ}$ para estas direções, analisando os dados em setores de $45^{\circ}$.

A variabilidade espacial e a anisotropia do teor de argila foram determinadas por meio do exame dos semivariogramas obtidos, ajustados a modelos teóri cos para a obtenção de seus parâmetros, tipo de modelo, pepita, patamar eal cance. Tais parâmetros, assinalados nos semivariogramas nesta mesma ordem, serviram de base para o cálculo de indicadores da anisotropia, além de serem aplicados diretamente na interpolação por krigagem no programa Surfer (Golden Software, 1995) para a geração do mapa de argila. A superfície gerada foi transportada para o programa Idrisi (Eastman, 1995), em que foi manipulada com segmentação em classes e pré-editada com adição de base geográfica.

A discussão dos resultados tomou como base as distâncias indicadas pelos semivariogramas correspondentes a quatro níveis de percentagem da variância (25, 50, 75 e 100\%). Com base nessas distâncias, foram calculados o número de amostras por área (e reciprocamente, a área de representatividade por amostra) e um fator proposto neste trabal ho para indicar o grau deanisotropia, chamado fator retangular ou de anisotropia. Estefoi definido como a proporção entre distâncias indicadas nos semivariogramas direcionais, em análises orientadas perpendicularmente entre si, para uma mesma percentagem da variância.

\section{RESULTADOS E DISCUSSÕES}

Os teores de argila variaram entre $180 \mathrm{e}^{2} 20 \mathrm{~g} \mathrm{~kg}^{-1}$, com média de $250 \mathrm{~g} \mathrm{~kg}^{-1}$ e coeficiente de variação de $14,5 \%$. O semivariograma obtido pela análise isotrópica para o teor de argila apresentou-se ajustado ao model o gaussiano, com val or de al cance ao redor de $170 \mathrm{~m}$ (Figura 1). O modelo gaussiano de semivariograma ajusta-se à variabilidade dos atributos da paisagem que variaram suavemente (Burrough, 1987), como as formas de relevo eo nível de água de subsolo, por exemplo. Portanto, o tipo de model o ajustado ficou coerente com a homogenei dade espacial dos Latossolos. Em Podzólico VermelhoAmarelo, solo menos homogêneo, de acordo com Al buquerque et al. (1996) e Salviano et al . (1998), o teor de argila superficial apresentou al cance ao redor de $40 \mathrm{~m}$, ajustando-se ao modelo esférico. Berg \& Klamt (1997) mostraram que os atributos facilmente modificados pel o manejo, tais como o teor de fósforo, pH e índice de saturação por base da camada superficial, apresentaram variabilidaderelativamente grande, quando comparados aos de outras, nas quais se inclui o teor de argila. 
Pelosemivariograma obtido por análise isotrópica, uma interpol ação passível de desvios correspondentes a $50 \%$ da variância total dos dados (F igura 1) teria de ser feita com não mais do que aproximadamente $80 \mathrm{~m}$ de distância entre amostras. Outros níveis de confiabilidade sãoatingidos com amostras espaçadas nas distâncias indicadas no eixo"h"dosemivariograma a partir da percentagem devariância da estimativa, indicada na escala à direita.

A comparação entre os semivariogramas direcionais indicou uma forteanisotropia, deacordo com a proporção entre os alcances da dependência espacial nos dois eixos (Figura 2). Como decorrência da continuidade do teor de argila ao longo da linha deplantio, o semivariograma ajustou-sea um model o gaussiano (Figura 2a). Em contraposição, o model o esférico foi mais bem ajustado para a variabilidade espacial dos dados no sentido da toposseqüência (Figura 2b). Observando o comportamento das duas curvas nas proximidades da origem ( $h=0)$, observouseligeira estabilidade inicial da semivariância para o primeiro caso, enquanto, no último, o crescimento da semivariância ocorreu desde a origem.

Os parâmetros da krigagem encontrados nos dois eixos de variação, segundo os modelos teóricos ajustados, foram comparados e analisados para a observação da anisotropia. O fator de anisotropia, calculado como a proporção entre os al cances, foi de $3,764 \mathrm{~m} \mathrm{~m}^{-1}$ para a direção da linha de plantio (ou, reciprocamente, $0,2657 \mathrm{~m} \mathrm{~m}^{-1}$ na toposseqüência). Semel hante comportamento foi observado nos resultados de teor de argila encontrados por Burrough (1987), com fator de anisotropia de aproximadamente $2 \mathrm{~m} \mathrm{~m}^{-1}$ (sob área de terraços aluviais, com teores de argila de 200 a $400 \mathrm{~g} \mathrm{~kg}^{-1}$ ). Este efeito acarretou uma configuração oval, ou anisotrópica, da área de influência dos dados amostrais, em conformidade com a estrutura da variabilidade espacial observada. A anisotropia deve ser atribuída a fatores intrínsecos ao solo,

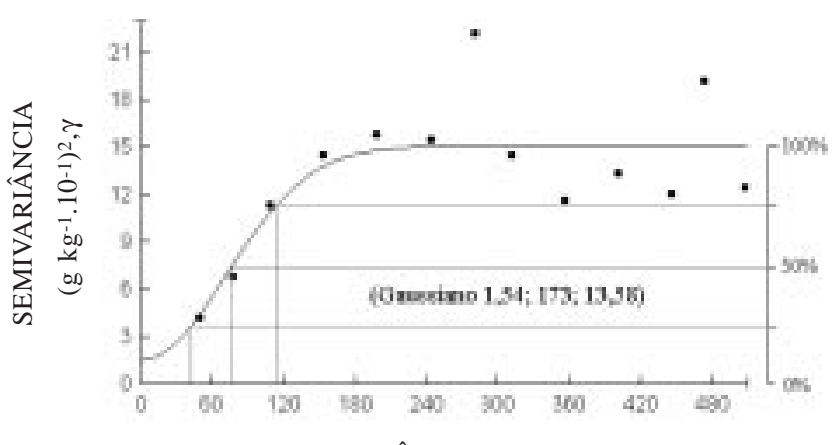

DISTÂNCIA, m, h

Figura 1. Semivariograma isotrópico para o teor de argila superficial do solo $(0-20 \mathrm{~cm})$. (Entre parênteses; modelo; efeito pepita; alcance e patamar).

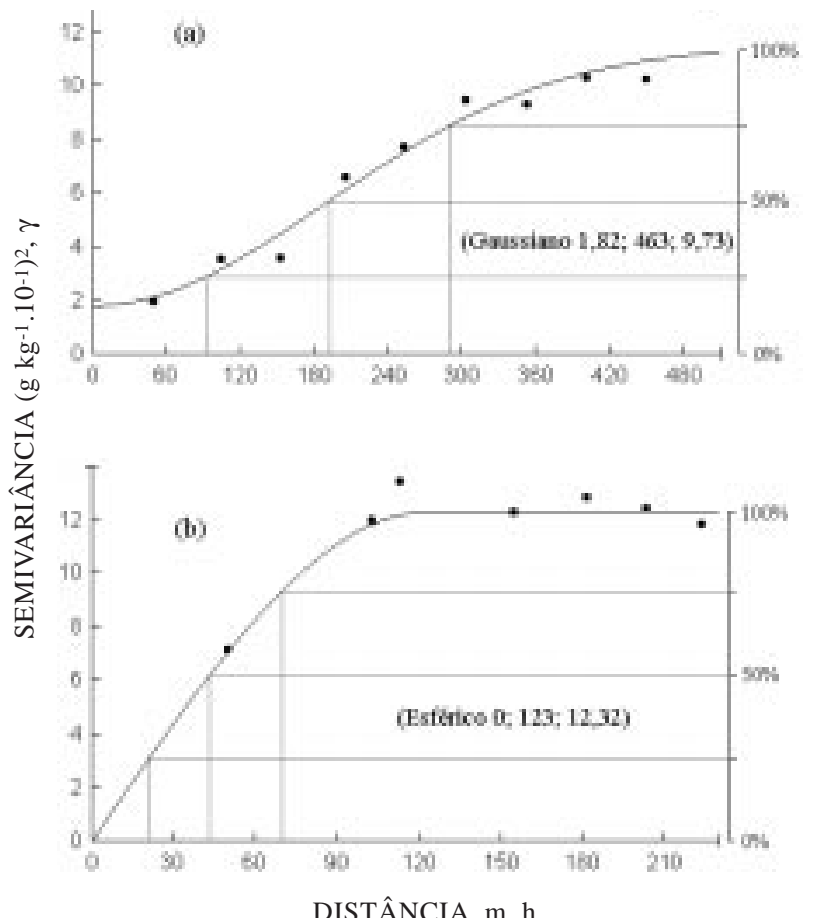

Figura 2. Semivariogramas para o teor de argila na camada superficial do solo nas direções (a) da linha de plantio e (b) da toposseqüência. (Entre parênteses; modelo; efeito pepita; alcance e patamar).

condicionados pela topografia no processo de formação, e extrínsecos, como a mecanização, di visão de gl ebas e demais práticas de manejo, que são feitas no sentido das linhas de plantio, transversalmente ao gradiente topográfico.

Os fatores de anisotropia com base no alcance foram associados aos respectivos conjuntos de parâmetros dos modelos de semivariogramas, com suas direções, no processo de krigagem anisotrópica. O mapeamento resultante mostrou-se mais coerente do que quando se aplicaram as mesmas técnicas sem considerar a anisotropia. As imagens apresentadas na figura 3 representam o mapa do teor de argila da camada superficial, segmentado em classes de variação segundo a legenda. A classificação destas áreas foi feita não sobre os dados de tradagem diretamente, porém sobre a superfície de variação contínua, por sua vez obti da pela interpolação destes dados. I sto permitiu que os limites entre as classes definidas fossem alocados adequadamente, com base na distribuição espacial do atributo avaliado. As manchas de classificação do solo sobre o modelo anisotrópico mostraram-se mais harmônicas com a toposseqüência, coalescendo preferencialmente áreas amostrais semelhantes que estivessem alinhadas na direção de menor variabilidade (ou maior dependência) espacial, ou seja, ao longo da 
linha de plantio. Sobre o modelo isotrópico, estas pequenas manchas foram classificadas como pontos isolados, sem coal escerem, ou foram engl obadas na classe majoritária circunvizinha.

Além da geração de mapas mais confiáveis, outro impacto importante das técnicas que levam em conta a anisotropia é a modificação do dimensionamento de parcelas amostrais, considerando as diferenças de al cance entreas direções anal isadas. Os desenhos de esquemas de amostragem e de procedimentos de espacial izaçãosãosubstancial mentefavoreci dos pelo estudo dos semivariogramas (Atkinson, 1996; Gonçalves et al., 1999). É esperado queo detalhamento da variabilidade espacial através de semivariogramas direcionais, quando pertinentes, traga ainda mais benefícios, uma vez que avalia e descreve a variabilidadecoma anisotropia, queéum componente importante da variação dos dados.

O quadro 1 resume as principais implicações geométricas sobre um plano de amostragem da área, considerando o efeito ani sotrópico. As dimensões das parcelas amostrais foram definidas pelos níveis de percentagem da variância da estimativa indicados pel os semivariogramas obtidos (Figuras 1 e 2 ). Com base nessas dimensões, a área de representatividade das amostras (ha/amostra) variou de um esquema a outro. Observando a proporção entre estas áreas, pode-se avaliar o impacto da anisotropia sobre o número de amostras requerido para cada nível de variância desejável.
Decorre da mel hor representação da variabilidade pelo modelo anisotrópico que as áreas de representatividade das amostras são maiores, otimizando o desenho experimental. Reciprocamente, para um mesmo nível de variância, tem-se sistematicamente um menor número médio de amostras requeridas por hectare. Observando os dados comparativos, verificou-se que a economia é maior nos casos em que se deseja (ou setolera) maior variância de estimativa. Para o caso daqueles que amostram o solo para a obtenção de dados independentes (variância acima de 100\%), a economia pode chegar a $47 \%$ de amostras. A economia de amostragem foi menor para o caso oposto, no qual a amostragem foi realizada para a interpolação dos dados, no sentido de explorar a dependência espacial. Estas vantagens requerem que a amostragem seja estruturada em grades retangulares em vez de grades quadradas, de modo que cada amostra corresponda a um retângul o cujas dimensões sejam proporcionais ao fator de anisotropia. Sobrepondo-se esses diferentes retângulos, projetados conforme a percentagem da variância da estimativa nos níveis 25, 50, 75 e 100\%, para um único ponto, obteve-se a figura 4.

$\mathrm{Na}$ figura 4, foram exemplificados um ponto amostrado (a) e os pontos b e c, como posições de referência para auxiliar as seguintes considerações: supondo-sea necessidade de um levantamento para posterior geração de mapa por meio de interpol ação,

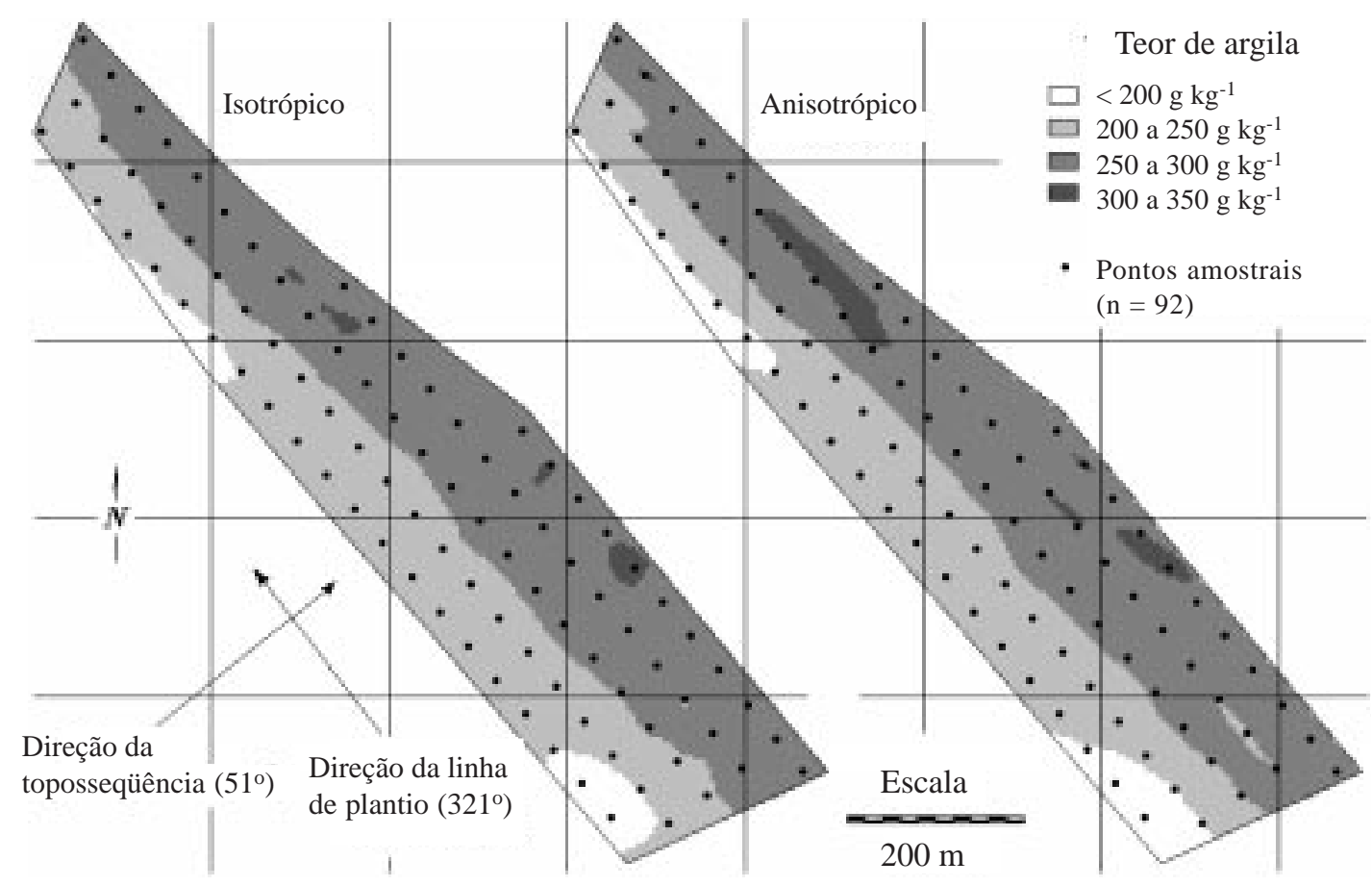

Figura 3. Mapas do teor de argila superficial, obtidos por krigagem com modelo isotrópico e anisotrópico. 
Quadro 1. Análise comparativa entre esquemas de amostragem isotrópica e anisotrópica

\begin{tabular}{|c|c|c|c|c|c|c|c|}
\hline \multirow{2}{*}{ Variância } & \multicolumn{2}{|c|}{ I sotropia } & \multicolumn{3}{|c|}{ Anisotropia } & \multicolumn{2}{|c|}{ Comparação } \\
\hline & Dimensão & Amostra & Dimensão & Amostra & Fator ret.(1) & Dens. Rel.(2) & E conomia ${ }^{(3)}$ \\
\hline$\%$ & $\mathrm{~m} \mathrm{~m}^{-1}$ & ha & $\mathrm{m} \mathrm{m}^{-1}$ & ha & & $\longrightarrow$ & 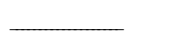 \\
\hline 25 & $40 \times 40$ & 6,25 & $95 \times 22$ & 4,7847 & 4,318 & 76,5 & 23,5 \\
\hline 50 & $80 \times 80$ & 1,5625 & $195 \times 43$ & 1,1926 & 4,535 & 76,3 & 23,7 \\
\hline 75 & $112 \times 112$ & 0,7972 & $287 \times 70$ & 0,4978 & 4,100 & 62,4 & 37,6 \\
\hline 100 & $173 \times 173$ & 0,3341 & $463 \times 123$ & 0,1756 & 3,764 & 52,6 & 47,4 \\
\hline
\end{tabular}

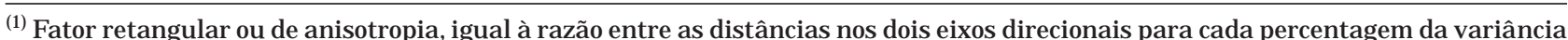
nos semivariogramas. ${ }^{(2)}$ Densidade Relativa de amostragem, ou a razão de amostras/ha no esquema anisotrópico em relação ao isotrópico. ${ }^{(3)}$ Economia da amostragem anisotrópica.

os pontos amostrados na vizinhança do ponto a devem estar a distâncias correspondentes a um nível pequeno de variância da estimativa. Observando o dimensionamento baseado no semivariograma isotrópico (quadrados, em cinza), o ponto b localizase dentro da área de variância de estimativa inferior a $50 \%$. Entretanto, constatada a anisotropia (retângulos, em preto), verifica-se que, naquela direção a-b (ou toposseqüência), o ponto b já está local izado na área com variância de estimativa acima de $75 \%$. Portanto, na direção da toposseqüência, os esquemas de amostragem isotrópicos tendem a subdimensionar a intensidade de amostragem.

As mesmas considerações podem ser feitas, para o caso de amostras compostas, as quais devem ser tomadas dentro das áreas delimitadas pel o nível de variância tolerado. Considerações recíprocas são feitas supondo-se que a amostragem seja delineada para fornecer dados independentes, ou seja, no caso de se procurarem pontos distanciados além do alcance (100\%). Uma análise isotrópica indicaria que o ponto c (Figura 4) está livre da dependência espacial em relação ao ponto a, conforme é desejável para este caso. Todavia, a anisotropia observada na análise indicou que, na realidade, o ponto c conserva mais do que $50 \%$ de sua dependência em relação ao ponto a, uma vez que a variância de estimativa naquela direção (direção da linha de plantio), àquela distância, está abaixo do limite de $50 \%$. Na direção transversal (toposseqüência), esta distância já seria suficiente para garantir a independência dos dados. Distribuindo o desenho das células amostrais da figura 4 em grades, pela área de estudo, obtêm-se proposições de amostragem (Figura 5) com níveis de variância de estimativa de 25, 50, 75 e $100 \%$ no esquema isotrópico e no anisotrópico.

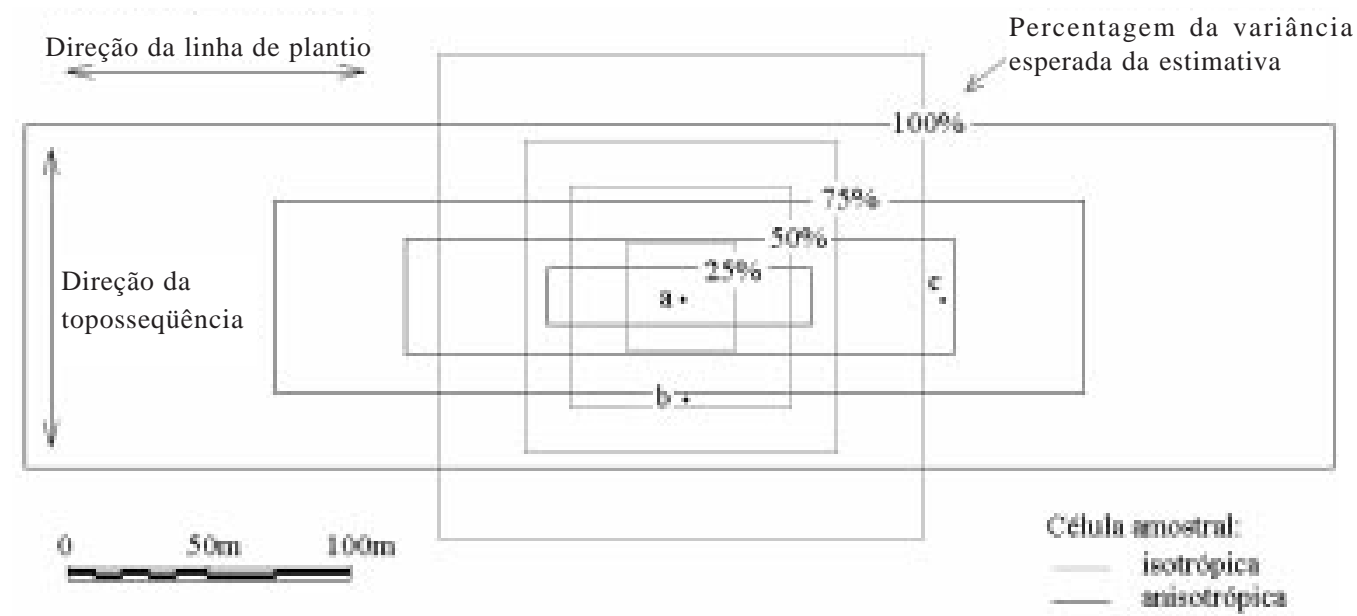

Figura 4. Redimensionamento da área de representatividade de amostras, considerando a variância esperada da estimativa, segundo os semi variogramas isotrópicos e ani sotrópicos. 
Os esquemas de amostragem anisotrópicos (Figura 5) reproduzem o princípio da descrição dos solos de uma unidade de paisagem através de transectos, que, apesar ser uma técnica comum de representação dos resultados de levantamentos pedológicos, não é levada em conta na fase de planejamento do trabal ho de campo. As vantagens advindas de tal prática envolvem a confiabilidade do mapa inter polado e a operacional idade (custo) do levantamento, de maneira interligada. A incorporação da anisotropia permite, dado um número possível de amostras (determinado por um recurso finito, por exemplo), fazer um mapeamento mais confiável, apenas redistribuindo os pontos de acordo com as dimensões anisotrópicas. Por outro lado, desejando atingir certo nível de confiabilidade, indicado pela percentagem da variância da estimativa, o esquema anisotrópico permite que se faça o levantamento com um menor número de pontos amostrais.

Os trabalhos de descrição da variabilidade espacial de atributos numéricos de solos devem incorporar ofenômeno da anisotropia. Caracterizando a anisotropia de diferentes variáveis, sob variadas condições (topografia, tipo de solo, histórico de manejo), os levantamentos a serem feitos poderão, já no planejamento, incluir este efeito, aproveitando, assim, as vantagens aventadas nesta pesquisa. Por exemplo, pode-se recomendar para as condições específicas deste trabalho, com segurança, que a distância entre amostras no sentido da toposseqüência seja 1/3 da distância entre amostras no sentido da linha de plantio, quando se quiser o mapeamento do teor de argila.
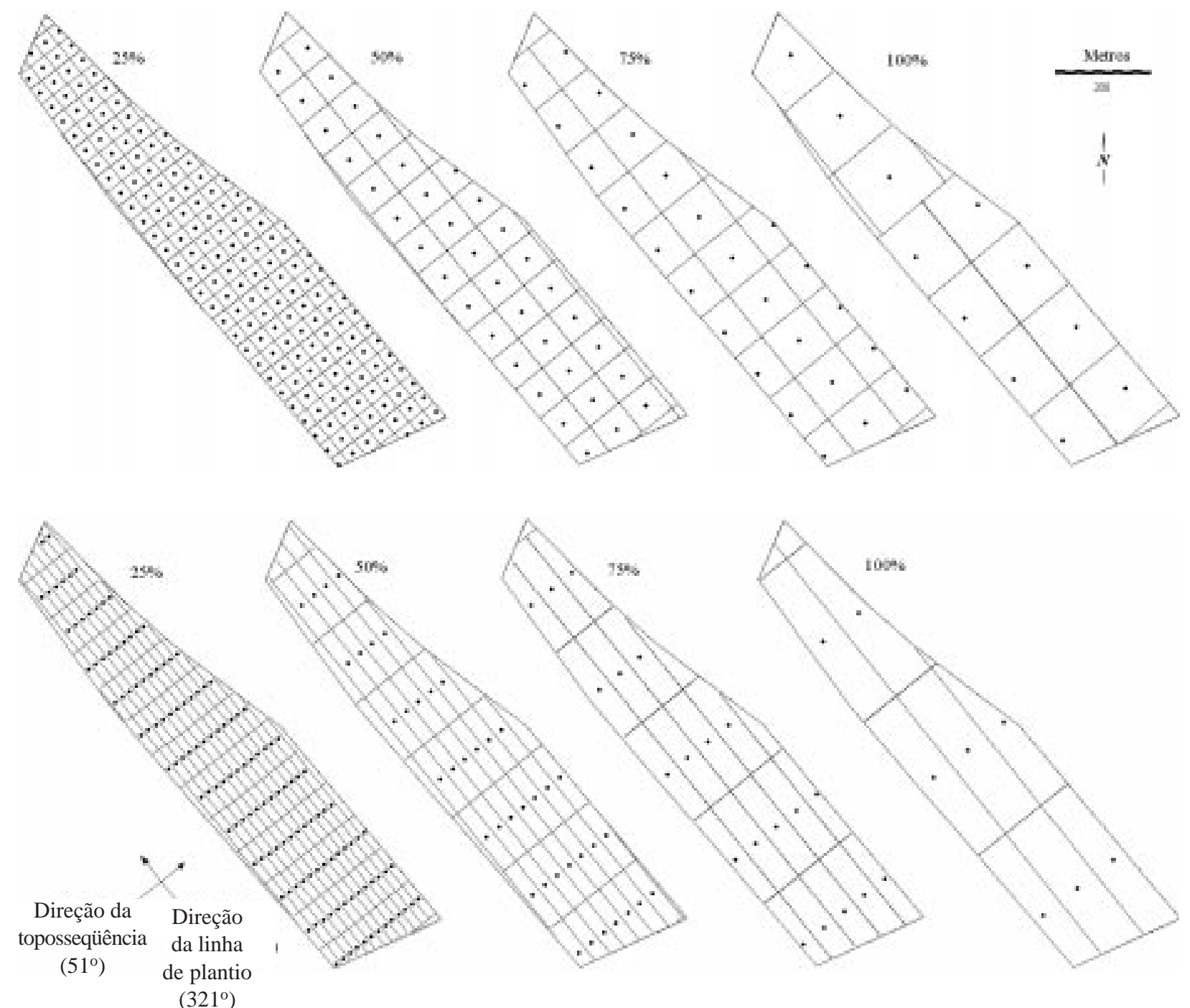

Figura 5. Proposições de amostragem sob níveis de percentagem da variância da esti mativa, segundo análise isotrópica (conjunto superior) e anisotrópica (inferior). 


\section{CONCLUSÕES}

1. O teor de argila do sol o mostrou-se fortemente anisotrópico, com maior variabilidade no sentido da toposseqüência; a proporção entre o alcance na direção de plantio (nível) e na direção da toposseqüência foi de aproximadamente $3,7 \mathrm{~m} \mathrm{~m}^{-1}$, correspondendo a uma configuração oval da área de influência de cada ponto amostral.

2. A classificação da superfíciecontínua (imagem) do atributo analisado mostrou-se uma técnica adequada de geração de mapas, por incorporar sua variabilidade na del imitação das classes de intervalo de variação. A interpolação por krigagem permitiu, ainda, quando evidenciados, considerar efeitos anisotrópicos.

3. A representação da variabilidade espacial por meio de um modelo anisotrópico subsidiou a concepção de esquemas de amostragem otimizados em relação à abordagem isotrópica. A reestruturação anisotrópica da grade de amostragem forneceu melhor relação entre a confiabilidade do levantamento e seu custo, representado pelo número de amostras.

4. O estudo da anisotropia é importante na descrição da variabilidade de propriedades dos sol os porque erros de desenho experimental eamostragem são cometidos quando estes são el aborados com base na descrição da variabilidade espacial, nos casos em que se ignoram possíveis efeitos anisotrópicos existentes.

\section{LITE RATURA CITADA}

ALBUQUERQUE, J.A.; REINERT, D.J . \& FIORIN, J.E. Variabilidade de solo e planta em Podzólico VermelhoAmarelo. R. Bras. Ci. Solo, 20:151-157, 1996.

ATKINSON, P.M. Optimal sampling strategies for raster-based geographical information systems. Global Ecol. Biog. Letters, 5:271-280, 1996.

BERG, M. van der \& KLAMT, E. Variabilidade espacial de características de sol os na região do planalto médio, RS: II . Análise da semivariância e da variância. R. Bras. Ci. Solo, 21:401-408, 1997.

BURROUGH, P.A. Spatial aspects of ecological data. In: J ONGMAN, R.H.; ter BRAAK, C.J .F. \& TONGEREN, O.F.R., eds. Data analysis in community and landscape ecology. Wagenigen, Pudoc, 1987. p.213-251.

CAMARGO, O.A.; KLAMT, E. \& KAUFFMAN, J .H. Classificação de solos usada em levantamento pedológico no Brasil. B. Inf. SBCS, 12:11-33, 1987.
CAMARGO, O.A.; MONIZ, A.C.; J ORGE, J.A. \& VALADARES, J.M.A.S. Métodos de análise química, mineralógica e física de solos do Instituto Agronômico de Campinas. Campinas, Instituto Agronômico de Campinas, 1986. 94p. (Boletim técnico, 106)

CARVALHO, O.S.; GASCÓ,J .M.; LOPÉZ, F.G. \& REQUEJ O,A.S. Variabilidade espacial de algumas propriedades químicas e físicas de um solo submetido a diferentes sucessões de cultivo. R. Bras. Ci. Solo, 22:497-503, 1998.

EASTMAN, J.R. Idrisi for Windows : user's guide. Worcester: Clark University, 1995. 440p.

EMPRESA BRASILEIRA DE PESQUISA AGROPECUÁRIA EMBRAPA. Centro Nacional de Pesquisa de Solos. Sistema Brasileiro de Classificação de Solos. Brasília. 1999. 412p.

ENGLUND, E. \& SPARKS, A. GEO-EAS (Geostatistical Environmental Assessment Software). User's Guide. Las Vegas, Environment M onitoring Systems L aboratory/Office of Research and Development. U.S. Env. Protection Agency, 1988. (EPA600/4-88/033)

GOLDEN SOFTWARE. Surfer Version 6.01- Surface Mapping System. Golden, Golden Software, 1995. não paginado.

GONÇALVES,A.C.A.; FOLEGATTI, M.V. \& VIEIRA, S.R. Padrões de amostragem e intensidade de krigagem na caracterização do armazenamento de água no solo, em área irrigada por pivôcentral. R. Bras. Ci. Solo, 23:485-495, 1999.

LEGENDRE, P. Spatial autocorrelation: trouble or new paradigm? E cology, 74:1659-1673, 1993.

OLIVER, M.A. \& WEBSTER, R. Krigging: a method of interpolation for geographical information systems. Int. J . Geog. Inf. Syst., 4:313-332, 1990.

PANNATIER, Y. VarioWin: Software for Spatial Data Analysis in 2D. New York, Springer-Verlag, 1996. p.316.

SALVIANO,A.A.C.;VIEIRA, S.R. \& SPAROVEK, G. Variabilidade espacial de atributos do solo e de Crotalaria juncea $L$. em área severamente erodida. R. Bras. Ci. Solo, 22:115-122, 1998.

SOIL SURVEY STAFF. Keys to Soil Taxonomy. 4.ed. Blacksburg, Virginia. 1990. (SMSS Technical Monograph, 6)

SOUZA, L.; COGO, N.P. \& VIEIRA, S.R. Variabilidade de fósforo, potássio e matéria orgânica no solo em relação a sistemas de manejo. R. Bras. Ci. Solo, 22:77-86, 1998.

SOUZA, L.; COGO, N.P. \& VIEIRA, S.R. Variabilidade de propriedades físicas equímicas do solo em um pomar cítrico. R. Bras. Ci. Solo, 21:367-372, 1997.

VALERIANO, M.M. Estimativa de variáveis topográficas por geoprocessamento para modelagem da perda de solos. Rio Claro, Universidade Estadual Paulista, 1999. 172p. (Tese de Doutorado)

VIEIRA, S.R. Curso de atualização em conservação de solos. Geoestatística em estudos de variabilidade espacial. Campinas, Instituto Agronômico de Campinas, 1995. 61p. (Apostila - parte I) 
